\title{
Ground Verification of the Feasibility of Telepresent On-Orbit Servicing
}

\author{
Enrico Stoll \\ Ulrich Walter \\ Institute of Astronautics (LRT) \\ Technische Unversität München \\ Garching, Germany \\ e.stoll@mytum.de \\ walter@tum.de
}

\author{
Jordi Artigas \\ Carsten Preusche \\ Philipp Kremer \\ Gerd Hirzinger \\ Institute of Robotics and Mechatronics \\ German Aerospace Center (DLR) \\ Oberpfaffenhofen, Germany \\ jordi.artigas@dlr.de \\ carsten.preusche@dlr.de \\ philipp.kremer@dlr.de \\ gerd.hirzinger@dlr.de
}

Jürgen Letschnik

LSE Space Engineering \& Operations AG

Wessling, Germany

juergen.letschnik@lsespace.com

\author{
Helena Pongrac \\ Human Factors Institute \\ University of the Bundeswehr \\ Neubiberg, Germany \\ helena.pongrac@unibw.de
}

\begin{abstract}
In an ideal case telepresence achieves a state, where a human operator can no longer differentiate between an interaction with a real environment or a technical mediated one. This state is called transparent telepresence. The applicability of telepresence to on-orbit servicing (OOS), i.e. an unmanned servicing operation in space, teleoperated from ground in real time, is verified in this paper.

For that purpose, a communication test environment was set up on ground, which involved the Institute of Astronautics (LRT) ground station in Garching, Germany and the ESA ground station in Redu, Belgium. Both were connected via the geostationary ESA data relay satellite ARTEMIS. Utilizing the data relay satellite, a teleoperation was accomplished, in which the human operator as well as the (space) teleoperator was located on ground. The feasibility of telepresent OOS was evaluated, using an OOS test bed in the Institute of Mechatronics and Robotics at the German Aerospace Center (DLR). The manipulation task was representative for OOS and supported real time feedback from the haptic-visual workspace. The tests showed that complex manipulation tasks can be fulfilled by utilizing geostationary data relay satellites.

For verifying the feasibility of telepresent OOS, different evaluation methods
\end{abstract}


were used. The properties of the space link were measured and related to subjective perceptions of participants, which had to fulfill manipulation tasks. An evaluation of the transparency of the system, including the data relay satellite, was accomplished as well.

\section{Introduction}

Spacecrafts are the only complex engineering systems without maintenance and repair infrastructure. Occasionally, there are space shuttle based servicing missions, starting with the Solar Maximum Repair Mission (SMRM) in 1984, but there are no routine procedures foreseen for individual spacecrafts. Most malfunctioning spacecrafts require only a minor maintenance operation on orbit, a so-called On-Orbit Servicing (OOS) mission, to continue operational work. Instead, they have to be replaced due to the lack of OOS opportunities. The accomplishment of OOS missions would, similar to terrestrial servicing procedures, be of great benefit for spacecraft operators, since a wide spectrum of use cases exists as e.g. spacecraft assembly, orbit transfer, maintenance and repair, resupply, or even safe deorbiting.

\subsection{Motivations}

Following the SMRM, there were several Space Transportation System (STS) based servicing missions (e.g. Intelsat VI (F-3) and the Hubble Space Telescope). These incipient OOS missions did not only demand a complex and cost intensive shuttle mission, but also the application of Extra-Vehicular Activities (EVA). Astronauts had to leave the safe environment of the space station in order to retrieve and repair the spacecrafts in outer space, only protected by their suits.

Based on the criticality of an EVA, concepts of robotic applications have been developed that can be controlled by astronauts. However, such devices like the Special Purpose Dexterous Manipulator (SPDM) by the Canadian Space Agency (Mukherji et al., 2001) or the robotic astronaut (Robonaut) (Peters II and Campbell, 1999), developed by the National Aeronautics and Space Administration (NASA), are in situ teleoperated by astronauts (from the Space Shuttle or the International Space Station) and demand still the use of an STS. 
In contrast to this manned OOS missions, there are options to accomplish OOS missions unmanned. As it will be seen in section 1.2, unmanned spacecrafts (servicer satellites) are foreseen to accomplish OOS operations at a target satellite. For that purpose the explorative and manipulative possibilities of robots will be exploited to dock the servicer satellite with the malfunctioning target satellite and execute complex operations, remotely controlled from ground.

While there is much research undertaken on spacecraft autonomy, there are only a few space projects considering a telepresent control of the spacecraft, which is of special interest for the work, presented here. A telepresent control includes a human operator in a ground station controlling the robotic application and receiving instantaneous (visual and haptic) feedback from the spacecraft to the actions. This work analyzes, whether the concept of telepresence (control) is applicable to OOS. For that purpose a test environment, which focuses on space applications in Low Earth Orbit (LEO) was developed and set up on ground.

OOS in LEO is a special problem, since direct contact between a ground station and the servicing spacecraft is only given in small time intervals. However, the feasibility of OOS operations is highly dependent on whether and how long a communication link between the controlling ground station and the servicer spacecraft can be established. Tab. 1 will clarify the reason. The space shuttle based OOS missions of the Hubble Space Telescope (HST) are listed. Each of them required several EVAs resulting in a total EVA time of more than 24 hours. An OOS mission, which is telepresently controlled from ground demands an equivalent amount of contact time.

\begin{tabular}{|l|l|l|l|}
\hline flight & year & number of EVAs & total EVA time \\
\hline \hline STS -61 & 1993 & 5 & $35 \mathrm{~h} 28 \mathrm{~min}$ \\
\hline STS -82 & 1997 & 5 & $33 \mathrm{~h} 11 \mathrm{~min}$ \\
\hline STS -103 & 1999 & 3 & $24 \mathrm{~h} 33 \mathrm{~min}$ \\
\hline STS -109 & 2002 & 5 & $35 \mathrm{~h} 55 \mathrm{~min}$ \\
\hline \hline
\end{tabular}

TABLE 1: Space Shuttle based Hubble Space Telescope OOS missions

Using direct communication in LEO would accrodingly require several weeks or a complex 
ground station network. Since the HST orbits the Earth at approximately $570 \mathrm{~km}, 4-8$ orbit revolutions per day exist, in which a human operator could steer a robotic servicer for maximum 10 minutes per orbit revolution (Lundin and Stoll, 2006). Thus, for accomplishing complex OOS operations, the use of geostationary satellites is proposed, which increases the mean acquisition time of the spacecraft in LEO up to more than 1 hour per orbit revolution. The use of geostationary data relay satellites in turn, increases the round trip delay of the signal, that is, the time between operator action and spacecraft feedback. The main goal of this work is to prove that the utilization of geostationary (GEO) data relay satellites for OOS is reconcilable with a telepresent control of the servicer spacecraft.

\subsection{State of the art OOS technology demonstrators}

This section gives a brief overview of OOS technology demonstrators that were brought to orbit, that are still orbiting Earth, or pending. The emphasize is hereby, placed on the communication architecture (direct or relayed contact) and the manner of control (telepresence or autonomy).

The first robot in space, which has been remotely controlled from ground, was the Robot Technology Experiment (ROTEX) aboard the space shuttle Columbia in 1993. The operational modes were tele-sensor-programming (learning by showing), automatic (pre-programmed on ground), and teleoperation on-board (an astronaut controlled the robot using a stereo monitor). Further, a teleoperation by a human operator from ground, using predictive computer graphics, was performed. (Hirzinger et al., 2004)

ETS VII is a Japanese Engineering Test Satellite (ETS) capable of demonstrating bilateral teleoperation in space (Imaida et al., 2004). The spacecraft, consisting of a pair of satellites, was launched in 1997. Autonomous capturing of the smaller target satellite, inspection procedures and a series of manipulation operations was demonstrated (Oda, 2000).

The Robotic Component Verification aboard the ISS (Rokviss) is a German space technology experiment, which was installed in 2005 outside the International Space Station (ISS) at the Russian service module (Landzettel et al., 2006). Rokviss is a two joint robotic manipulator, 
controlled by a human operator via a direct radio link from the ground station in Weilheim, Germany (Preusche et al., 2006).

The Experimental Satellite System 10 (XSS-10) (Davis, 2005) was developed by the US Air Force. The space mission was launched in 2003 and the mission objectives included autonomous navigation and proximity operations.

The Experimental Satellite System 11 (XSS-11) (AFRL, 2007) was a micro satellite of approximately 100 kilograms. Launched in 2005, XSS-11 has been designed for testing autonomous technologies necessary for the inspection of malfunctioning satellites.

All operations of the Demonstration of Autonomous Rendezvous Technology (DART) (Rumford, 2003) mission were developed to be autonomous. Launched to verify hardware and software for rendezvous and proximity operations, the main objectives were the demonstration of station keeping and collision avoidance maneuvers. However, when DART approached the target, it overshot an important waypoint, and thus, the pre-programmed transition to the target satellite, and collided with it. A premature retirement of DART was the consequence. The mission plan of Orbital Express (BOEING, 2007) foresaw the validation of software for autonomous mission planning, rendezvous, proximity operations, and docking. Further tests of robotic OOS scenarios included fuel and electronics transfer, deployment of, and operations with a micro-satellite. The Swedish Space Cooperation (SSC), together with KayserThrede, Germany and Sener, Spain is developing the SMART Orbital Life Extension Vehicle (SMART-OLEV) (Tarabini et al., 2007). It aims at extending the operational life time of geostationary satellites.

The Deutsche Orbitale Servicing Mission (DEOS) (Sommer, 2003) will demonstrate diverse OOS scenarios such as rendezvous, inspection, formation flight, capture, stabilization, and controlled de-orbiting of the target and servicer compound. In this connection two modes for commanding the servicer are foreseen. On the one hand, there will be active ground control via telepresence, i.e. a control with instantaneous feedback to the human operator. On the other hand, it will also be possible to passively monitor autonomous operations from ground. The ranger robotics program started in 1992 as the Ranger (Parrish and Akin, 1996) Telerobotic Flight Experiment (RTFX) at the University of Maryland, USA. The goal was to 
develop a dexterous extravehicular space telerobot with four robot manipulators and a freeflight capability in space. In 1996 the program got redirected as a shuttle launch payload to the Ranger Telerobotic Shuttle Experiment (RTSX), was financed till 2001, and never advanced from an engineering model.

The Spacecraft for the Universal Modifications of Orbits (SUMO) (Bosse et al., 2004) is a project by the Naval Research Laboratory and funded by DARPA. The main goal is to develop a spacecraft, capable of demonstrating future OOS technologies. A series of autonomous rendezvous, grapple, and servicing experiments are planned.

Fig. 1 shows a possible classification of the OOS demonstrators. The classification is based on two criteria. Firstly, the options for communication with the OOS demonstrator (direct or relay to LEO or direct to GEO)in the respective orbit are considered. Secondly, the round trip delay between operator command and received feedback is considered. Since a telepresent operation demands an instantaneous feedback to the operator's action, different natures of round trip delay are defined. They are a first indication for the telepresence capability of the system.

The ideal or transparent telepresence (TTP) is, encouraged by the Rokviss experiments, here defined as being in the magnitude of approximately up to $0.1 \mathrm{~s}$. This allows the user to obtain instantaneous feedback of the telecommands, and technical means will enable the human operator to feel present in the removed environment. It is followed by telepresence (TP), being in the magnitude of approximately up to $1.0 \mathrm{~s}$. This is of special interest when considering applications, which are either located in GEO or of which the communication has to be relayed via the GEO. The large physical distance, which the communication signal has to bypass in both cases, causes a minimum round trip time larger than $0.24 \mathrm{~s}$ and $0.48 \mathrm{~s}$, respectively. Therefore, the round trip delay can be found in the telepresence branch. Transparent telepresence cannot be achieved for GEO or relay to LEO applications as emphasized in Fig. 1.

The third branch of the round trip delay criteria, covering round trip delays of approximately up to the $10.0 \mathrm{~s}$ magnitude, is defined as telerobotics (TR). This definition, encouraged by the ROTEX experiment, specifies an operation, in which the operator has to cope with 
comparable large time delays. While working in a virtual reality with a 3D model of the real environment, the human operator receives instantaneous simulated (predicted) feedback to the actions while they are executed in space a few seconds later and synchronized with the virtual reality afterwards. It is evident, that this approach is not usable for applications in which the environment is not sufficiently known, i.e. for an application, of which either the 3D model or its dynamics is not known in detail.

Autonomy and supervisory control schemes, opposing direct telepresence, are considered as a field where the round trip delay is not of importance since the operator on ground is not actively involved in the operations.

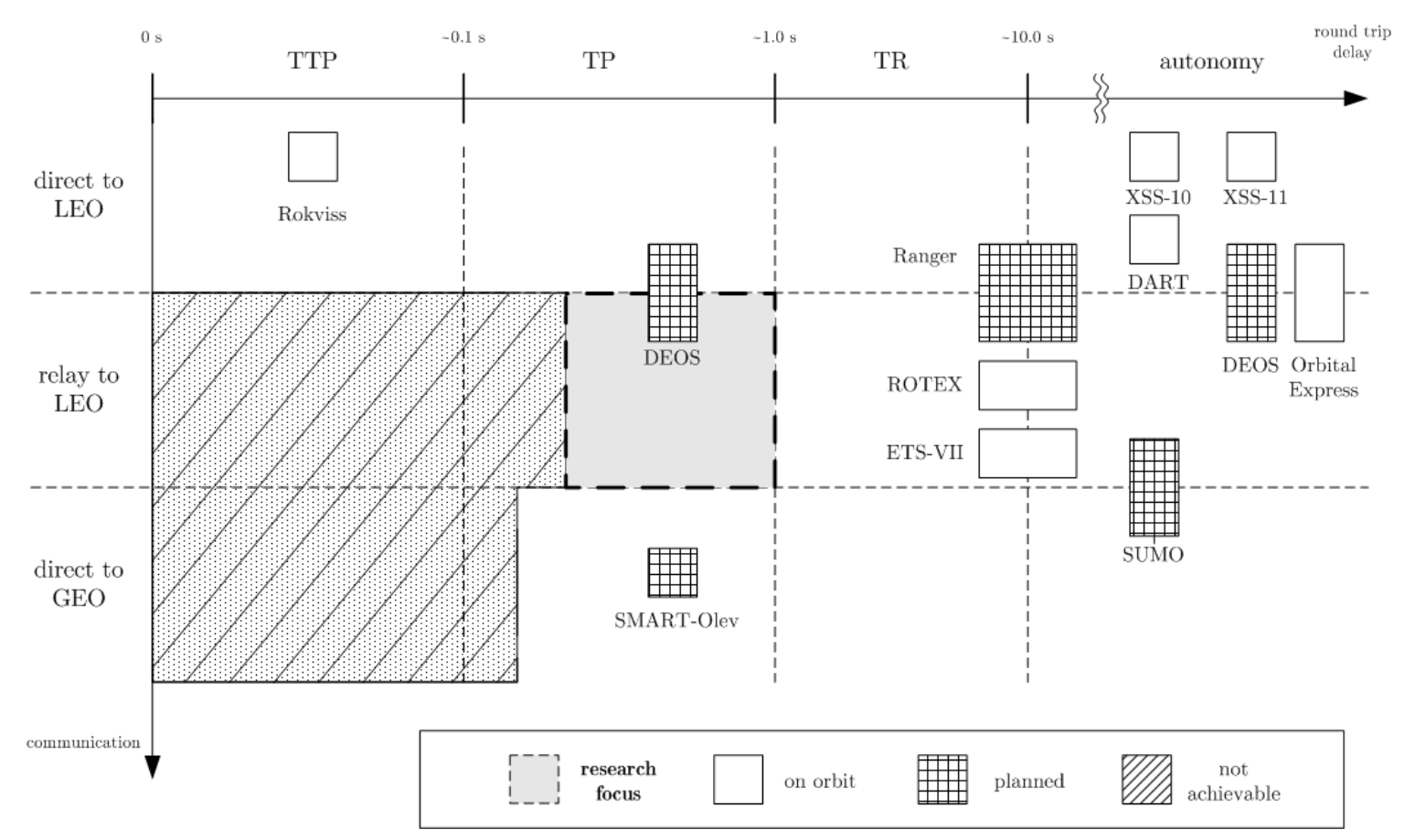

FIGURE 1 - A classification of OOS demonstrators

\subsection{Work overview}

As Fig. 1 shows, most OOS demonstrators, which are already on orbit utilized either the concept of TR or autonomy. Both are for the addressed reasons not reconcilable with the concept of telepresence. The preconditions for TTP do not hold for relayed communication, which in turn is necessary for complex and time demanding operations in LEO. This work considers the telepresence branch in connection with a relay approach as labeled in Fig. 1. 
To the authors' knowledge, no telepresence control with haptic feedback was executed via a geostationary relay satellite before. Thus, the work presents field experiments on Earth that demonstrate a telepresent teleoperation to a servicer in LEO. For that purpose a test environment was implemented, which included the ground station of the Institute of Astronautics (LRT) at Technische Universität München, Germany and the ground station of the European Space Agency (ESA) in Redu, Belgium. Both were connected via the ESA geostationary relay satellite ARTEMIS to set up a realistic environment. The robotic teleoperation, which was executed, comprised an OOS test bed developed by the Institute of Robotics and Mechatronics at the German Aerospace Centre (DLR) in Oberpfaffenhofen. Section 2 deals with the communication architecture of the test environment, including the geostationary relay satellite. Section 3 describes in detail the robotic OOS test bed. Section 4 depicts performance and results of the experiments and clarifies how telepresence or the degree of telepresence can be evaluated. Section 5 closes the treatment of the ground verification of telepresence for OOS with concluding remarks and considers future direction for continuing research.

\section{The communication architecture}

For verifying the feasibility of telepresent OOS in LEO, i.e. a telepresent servicing with fast feedback and relayed communication, a representative test environment was setup on ground. For that purpose the two ground stations in Germany and Belgium were connected via the GEO ESA relay satellite ARTEMIS.

\subsection{The geostationary relay satellite ARTEMIS}

The Advanced Relay Technology Mission (ARTEMIS) (Moens et al., 2003) was chosen to be the primary connector between human operator and teleoperator, both located on ground. ARTEMIS is the first European relay mission. It aimed at demonstrating new telecommunication techniques for data relay and mobile services. Being launched in 2001 and designed 
for 10 years, it is now situated at approximately $21.45^{\circ} \mathrm{E}$ and is able to provide service to Africa and Europe.
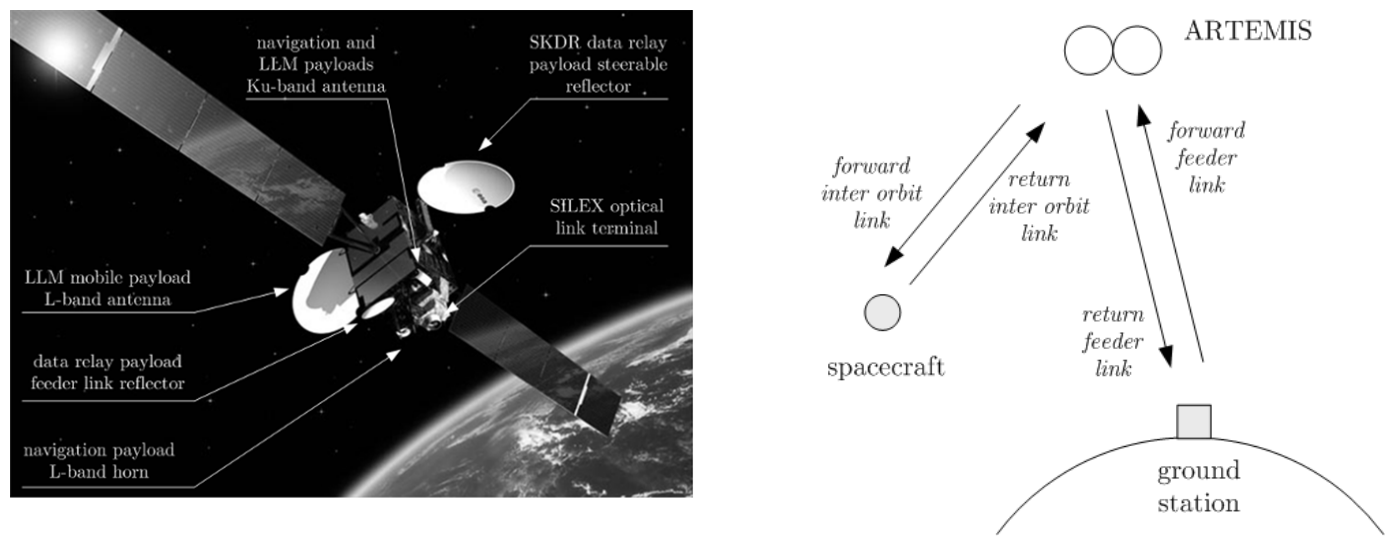

FIGURE 2 - ARTEMIS and its antennas (Moens et al., 2003) (left) and ARTEMIS link definitions (right)

Besides an optical link terminal, an L-band payload and a Ku-band payload as depicted in Fig. 2 (left), ARTEMIS features an S-and K-band Data Relay (SKDR) payload.The latter was in the focus of this work since the already existing LRT ground station utilized the S-band frequency range. Using the SKDR payload, ARTEMIS communicates with its supporting ESA ground station in Redu, Belgium. Thereby, the feeder links (forward / return) are in Ka-band and in K-band, respectively. The link definitions can be found in Fig. 2 (right).

In forward direction ARTEMIS can only provide one inter orbit link (IOL) at one time, i.e. either S-Band or Ka-Band communication is supported. In contrast, in return direction up to four IOLs can simultaneously be supported. Three of these channels can be allocated in K-band, while the fourth channel supports the S-band frequency range. Accordingly, ARTEMIS cannot provide data relay between two communication end points, only using S-band. The feeder link (FL) only works in K(a)-band. This fact is very important for the development of the communication architecture, which follows in the next section. 


\subsection{The mirror approach}

The ARTEMIS transponder specifications given in the previous section yielded very specialized test setup of the telerobotic test environment (Stoll, 2008). The FL as well as the IOL of ARTEMIS had to be utilized since ARTEMIS cannot operate the IOL only. The basic idea was to use both the ARTMEMIS FL and the IOL for communication to ground. The Institute of Astronautics operates a ground station in the S-band frequency range, which is suitable for communicating via the IOL with ARTEMIS; that is, it represents the spacecraft $(\mathrm{S} / \mathrm{C})$ in orbit. Further, the ESA ground station in Redu and its K(a)-band equipment can be used to establish the FL, as depicted in Fig. 3(left). The human operator (HO) send telecommands (TC) via the data relay satellite (DRS) ARTEMIS to the teleoperator (TOP), situated in the LRT ground station (GS). After being executed, sensor data of the TOP is being transmitted as telemetry (TM) back to the OP.

This communication architecture is not suitable for fundamental telepresence experiments,

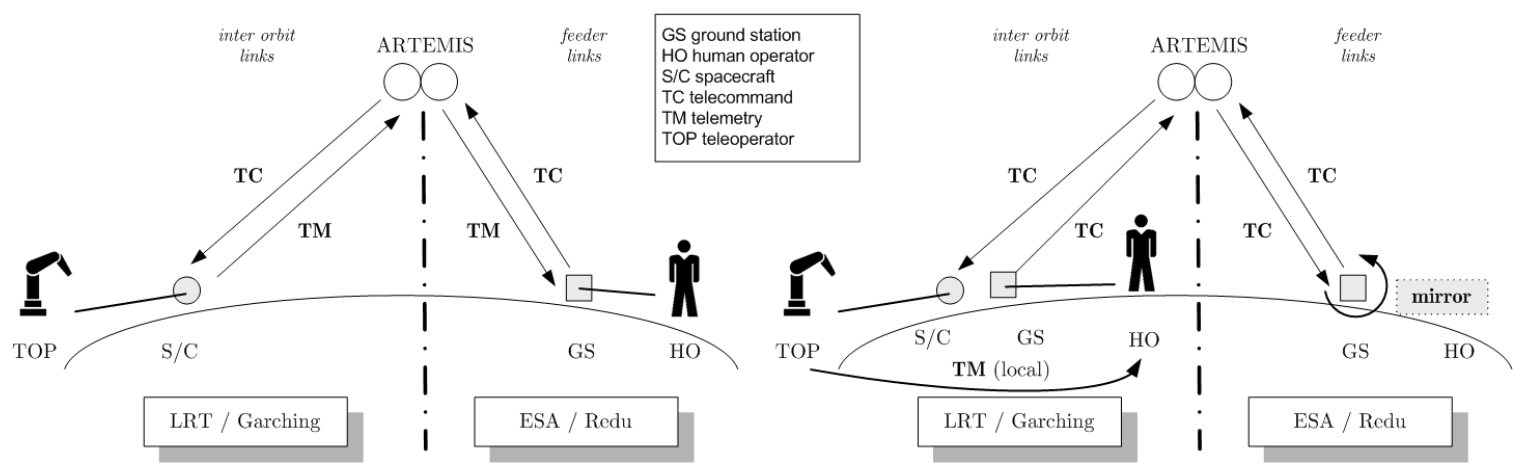

FigurE 3 - Communication architecture : (left) ideal and (right) using the mirror approach

as there is a large spatial distance between human operator in Garching (near Munich), Germany and teleoperator in Redu, Belgium. This causes an increase of system complexity. Therefore, the existing experimental system was modified.

The selected communication architecture featured a so-called signal (radio frequency) mirror as Fig. 3 illustrates. All available radio links were used for transmitting TC data after it is generated by the HO located in Germany. That way it was possible to locate the TOP in Germany as well. That way the mirror was introduced in the Redu GS, which is basically 
a local bypass at the communication system (in particular the IMBU (see Sec. 2.3). This means that the TC signal is reinjected immediately after reception into the uplink chain unchanged. Four hops ${ }^{1}$ are used for the TC before the TOP receives and processes it. The control loop between HO and TOP is closed locally (using TM data) with only one hop, which features a negligible time delay compared to the length of the TC hops. Thus, the round trip delays, which the operator perceives, are identical for the ideal communication architecture and the mirror approach.

The advantage of this mirror approach is evident. The LRT ground segment can be used as the GS and the $\mathrm{S} / \mathrm{C}$ of the experiment. Identical communication equipment can be used for both, since the same communication path (the IOL), with one frequency each for up- and downlink, is utilized. Further, no complex equipment, which has to be remotely configured, had to be installed at Redu.

\subsection{The communication setup}

As Fig. 3 shows, the LRT ground segment has to feature all functionalities of a ground station, as well as of the spacecraft. This means that in forward and return direction (almost) identical parameter (modulation, packet length et cetera) for configuring the communication link had to be used.

Traditional space missions show in contrast a very asymmetrical behaviour considering downlink and uplink capacity. TM and TC data, usually being in the range of a few kilobits per second (kbps), is transmitted using a narrowband. Additionally, the data acquisition of a satellite payload (e.g. synthetic aperture radar applications for Earth observation) may necessitate the utilization of a broad band with the capability to download several Megabits per second (Mbps). Tab. 2 exemplarily shows typical maximum uplink / downlink data rates of space systems. Rokviss is an example, which indeed shows the asymmetry, but not to such a degree as for example Cryosat. The reason is that the robotic TC data has to be sent at a very high sampling rate.

For the mirror approach experiments, presented here, this asymmetry had to be repealed.

1. In computer or communication networks one hop is the path between two communication nodes, e.g. between routers on Earth or between a S/C and a GS, when considering space applications. 
Uplink and downlink data rate are of the same value since they carry identical data. The haptic-visual feedback was realized locally, in order to not add delay.

\begin{tabular}{|l|r|}
\hline Satellite & max. uplink / downlink \\
\hline \hline Terrasar-X & $4 \mathrm{kbps} / 300 \mathrm{Mbps}$ \\
\hline Cryosat & $2 \mathrm{kbps} / 100 \mathrm{Mbps}$ \\
\hline SMOS & $4 \mathrm{kbps} / 18,4 \mathrm{Mbps}$ \\
\hline Rokviss & $256 \mathrm{kbps} / 4 \mathrm{Mbps}$ \\
\hline \hline
\end{tabular}

TABLE 2: Maximum data rates in uplink / downlink of exemplary space missions

Hence, an Integrated Modem and Baseband Unit (IMBU), as the core element of the communication, was custom made by Satellite Services BV for the specific requirements to the test environment. Fig. 4 shows a basic block diagram of the communication architecture. The DLR Institute of Robotics and Mechatronics, the LRT mission control centre, and the LRT ground station are highlighted. Transmitting and receiving telepresence data at the

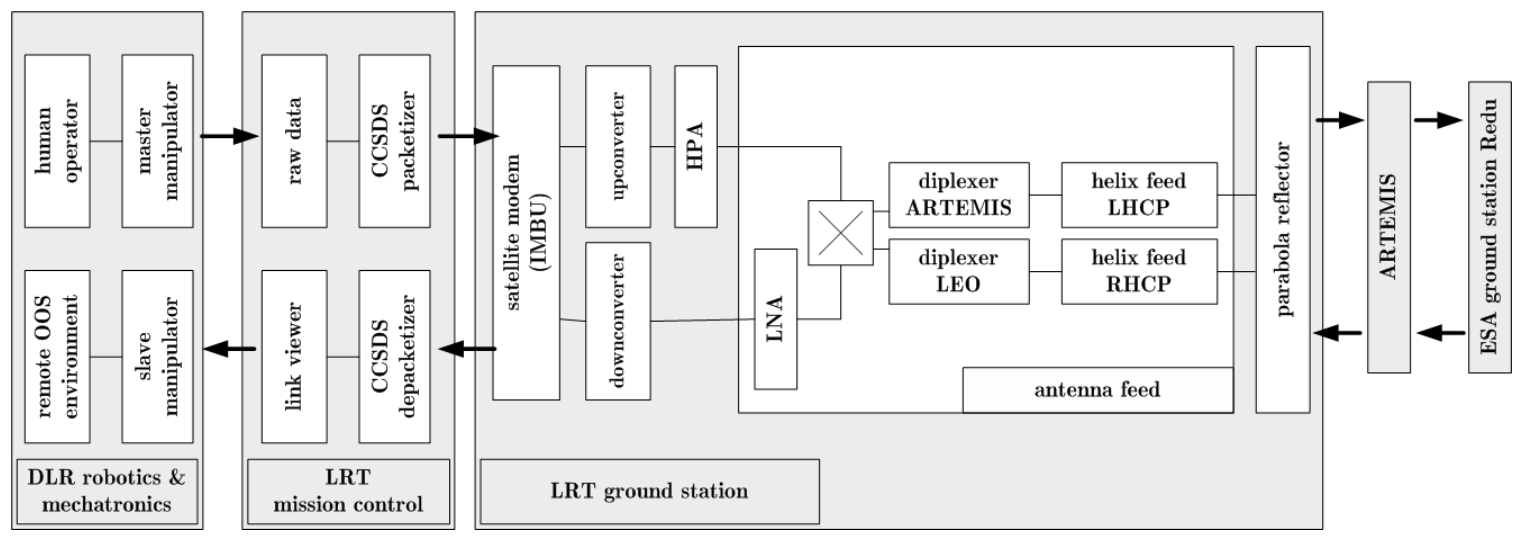

FIGURE 4 - The communication setup of the telerobotic test environment

LRT ground station was realized by utilizing specialized radio frequency up- and downconverter in the S-band frequency range. The converters feature an adjustable oscillator, with which the received or transmitted signal was mixed. This guaranteed a conversion between the intermediate frequency $(70 \mathrm{MHz})$, which the Integrated Modem and Baseband Unit (IMBU) utilized and the frequencies of the receiver and the transmitter, respectively.

As seen in Fig. 3 the ARTEMIS IOL forward and return links were used for S-band 
communication with the LRT ground station. Thus, frequencies had to be coordinated with the German federal network agency. The frequencies that have been allocated for the ARTEMIS service were 2076.5 MHz for IOL return (ground station uplink) and 2255.0 $\mathrm{MHz}$ for IOL forward (ground station downlink). Since these frequencies are usually used for communication in orbit, they were not usable on Earth without further regulations. Thus, the federal network agency allocated these frequencies only temporarily to LRT in the framework of an experimental radio communication license.

Broadband power amplifiers (High Power Amplifier / HPA, Low Noise Amplifier / LNA) had to be implemented into the test environment. The HPA was crucial for amplifying the upconverter signal to $20 \mathrm{~W}$. This transmitting power results from the link budget and ensures that the requirements of an amplification of minimum $+53.0 \mathrm{~dB}$ and a power output (at $1 \mathrm{~dB}$ compression point) of minimum $+43.0 \mathrm{dBm}$ were met. An according amplifier was not available off-the-shelf and was custom-built by MITEQ.

Further, the antenna feed system (see Fig. 4) had to be modified for the experiments since it had not only to support the communication with ARTEMIS in GEO, but also conventional satellites in LEO. A flexible polarization switch was implemented into the setup. It allowed switching between the left handed circular polarization (LHCP) of the IOL frequencies and the right handed circular polarization (RHCP), which is used for ground to satellite communication. A gain to noise ratio $G / T>4 \mathrm{~dB} / \mathrm{K}$ was realized by the setup and was sufficient for the telepresence experiments via the DRS.

ARTEMIS is a transparent satellite, i.e. received data is transmitted immediately after a frequency conversion. In contrast to so-called bent pipe technology. There is no demodulation, decoding, data correction, coding and modulation done as for regenerative satellites. This makes the ARTEMIS less flexible compared to regenerative satellites, but results in less processing time of data and accordingly supports the telepresence requirements of minimum round trip delays. 


\section{The bilateral control architecture of the OOS test bed}

A test bed for telepresent OOS has been coupled to the LRT mission control center as illustrated in Fig. 4. Located in the DLR institute of Robotics and Mechatronics in Oberpfaffenhofen, Germany, the test bed comprises two DLR light weight robots (LWR) as a bilateral (force coupled) master-slave system, and a target satellite dynamic emulator with an operations platform based on another LWR (Artigas et al., 2006).

As seen in Fig. 5 a 7DoF LWR-III is used as haptic man-machine interface. Further, a head mounted display with stereo visualization is used to obtain an immersive implementation of the visual channel. A DLR LWR-II is used as teleoperator robot which has been further equiped with an industrial gripper system in order to interact with the environment.

The OOS test bed has been designed to analyze different bilateral control strategies to cope with the presence of different varying round trip delays. The remote environment demonstrates a non-cooperative, malfunctioning satellite emulated by means of another LWR-II with a satellite test board attached to the end-effector of the robot. Featuring a deficiently working attitude control system, the satellite is tumbling in orbit and the operator has to fulfill two major tasks :

a) Docking : The satellite platform is to be caught by means of the gripper. Once the task is achieved, the target satellite is considered to have no relative motion to the servicer satellite.

b) Servicing : A series of servicing possibilities are given on target satellite side, which the operator has to solve. The manipulation of a bayonet nut connector and a series of cables is foreseen.

The motion and force commands are measured and transmitted via the data relay satellite to the distant slave manipulator. This in turn tracks the commands of the master manipulator and feeds the interaction forces with the remote environment back to the master manipulator system. The human operator is thus energetically coupled to the remote environment through the electromechanical elements. 

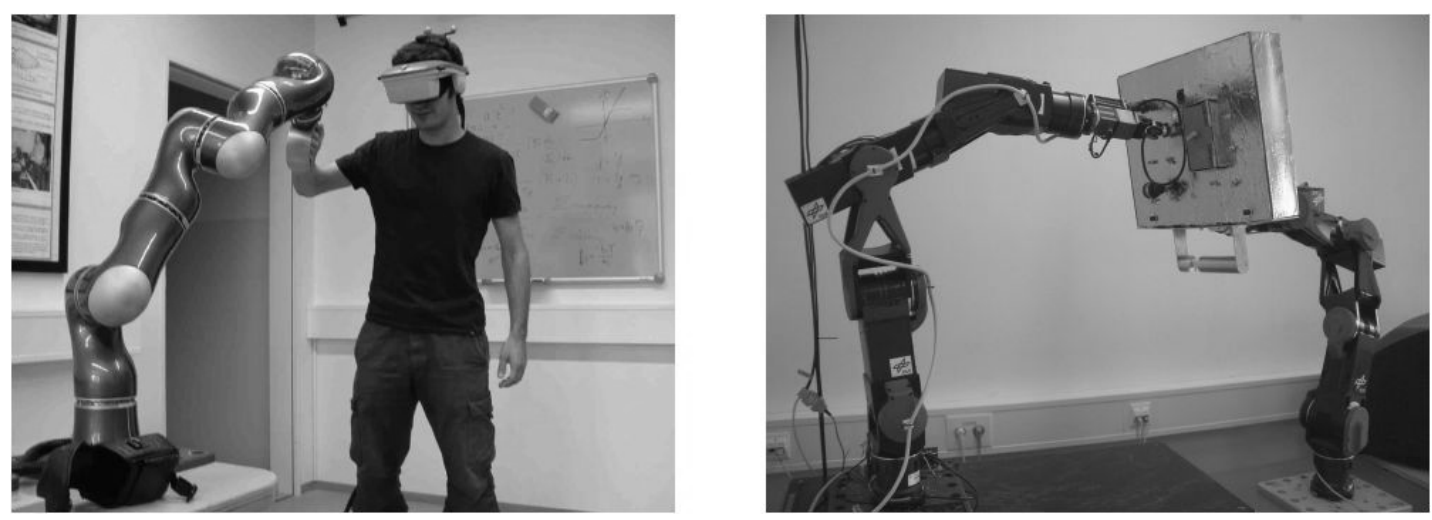

FIGURE 5 - DLR OOS Test bed

The inclusion of the human operator in the closed loop and the presence of the varying and comparably large time delay in the communication channel represents a challenging task. Most approaches dealing with time delayed haptic telepresence describe the system by means of power network elements, which are either designed to be passive (Anderson and Spong, 1989; Niemeyer, 1996) or will be adaptive in nature to keep passivity on the time domain (i.e. passivity not as a design constrain (Hannaford and Ryu, 2000; Artigas et al., 2007; Artigas et al., 2008)).

One of the most remarkable approaches is the classical method of the Scattering transformation or its Wave Variables formulation introduced in (Anderson and Spong, 1989; Niemeyer, 1996). By using the electrical-mechanical analogy, the wave variables transformation uses the power conjugated variables of force and velocity to define wave variables, the same way voltages and currents are related to energy waves in transmission lines. A wavy system has the interesting feature that passivity is preserved in the presence of time delay. Thus, the two-port network created by a communication channel with time delay described in terms of wave variables is a passive system which will not alter stability for any amount of constant time delay.

The bilateral control method used here is based on the wave variables approach. The next subsections review the wave transformation and its passivity aspects, and introduces a method to cope with the variation of the delay and the package loss. 


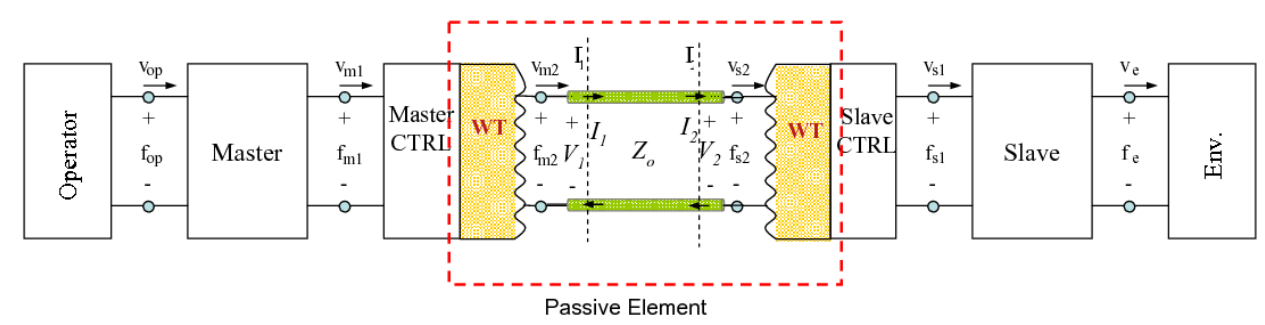

FiguRE 6 - Wave-based position-force teleoperation control scheme.

\subsection{Wave variables}

Wave variables present an extension to the theory of passivity and are based on the concepts of power and energy. The transformed variables, $u$ and $v$, are an algebraic relation of the power conjugated variables $\dot{x}$ and $F$, velocity and force respectively. $u$ is the wave variable traveling from master to slave. $v$ is the returning wave, from slave to master.

\subsubsection{Position-Force system with constant time delay}

Fig. 6 shows a wave variables -based position-force scheme. Two PI controllers at master and slave sites are responsible for minimizing the end effector position errors between master and slave, hence generating the required manipulation force on slave site and haptic interaction forces on master site. Note that the channel is isolated from the rest of the system by means of the wave transformers on each side of the channel, which in turn separate power variables domain from wave domain. The wave transformation equations for this scheme are (1) for master site and (2) for slave site, denoted by $m$ and $s$, respectively. Here $u$ represents the wave, sent from the master to the slave and $v$ the returning wave from the slave to the master.

$$
\begin{aligned}
& u_{m}(t)=\frac{b \dot{x}_{m d}(t)+F_{m}(t)}{\sqrt{2 b}}, v_{m}(t)=\frac{b \dot{x}_{m d}-F_{m}}{\sqrt{2 b}} . \\
& u_{s}(t)=\frac{b \dot{x}_{s d}(t)+F_{s}(t)}{\sqrt{2 b}}, v_{s}(t)=\frac{b \dot{x}_{s d}(t)-F_{s}(t)}{\sqrt{2 b}} .
\end{aligned}
$$

Here $b$ is the wave impedance constant, $F_{m}$ and $F_{s}$ are local controller force commands and $\dot{x}_{m d}$ and $\dot{x}_{s d}$ are desired motion velocities of the master and slave respectively. 
For the complete wave formulation please refere to (Niemeyer, 1996).

\subsection{Passivity condition}

Defining $P_{i n}$, the power entering a system, as the scalar product between input vector $x$ and output vector $y$, and $E_{\text {store }}$ as the stored energy of the system, the system is passive if and only if

$$
\int_{0}^{t} P_{\text {in }} \mathrm{d} \tau=\int_{0}^{t} x^{T} y \mathrm{~d} \tau \geq E_{\text {store }}(t)-E_{\text {store }}(0) .
$$

Without loss of generality, it can be assumed that the initial stored energy in the channel is equal to zero, $E_{\text {store }}(0)=0$. Further the forward and backward delays are assumed to be constant as a first estimation. Then the shift of wave signal can be formulated as follows.

$$
u_{m}\left(t-T_{f w d}\right)=u_{s}(t), \quad v_{m}(t)=v_{s}\left(t-T_{b w d}\right) .
$$

In the wave domain, condition (3) leads to

$$
\frac{1}{2}\left[\int_{t-T_{f w d}}^{t} u_{m}^{2}(\tau) \mathrm{d} \tau+\int_{t-T_{b w d}}^{t} v_{s}^{2}(\tau) \mathrm{d} \tau\right] \geq 0,
$$

which holds true as long equation (4) (constant time delay and guarantee of signal delivery) does. Accordingly, the stability of the teleoperator system is guaranteed.

\subsubsection{Varying time delay and package loss}

As previously seen in Chapter 2 the radio link from Garching - ARTEMIS - Redu imposes a set of characteristics which produce a direct impact upon the bilateral control. The previous section assumed an ideal delayed communication, with a constant time delay and zero package loss and bit error rates. In real scenarios, however, the delay cannot be considered constant and considerable package loss and bit error rates will be presented.

UDP is used for network connection of LRT and DLR due to its fast exchange rate (the smaller the delay, the better the operation performance and transparency), but this protocol is subject to packet drops and reordering because the transmission is not controlled in the network layers. 


\section{Time-varying delay}

Let forward and backward delays by defined by the time functions $T_{f w d}(t)$ and $T_{b w d}(t)$. Equation (4) can be reformulated :

$$
u_{m}(t)=u_{s}\left(t+T_{f w d}(t)\right), v_{m}\left(t+T_{b w d}(t)\right)=v_{s}(t)
$$

$T_{f w d}(t)$ and $T_{b w d}(t)$ functions are unknown at time $t$ for the master and slave sent waves $u_{m}(t)$, $v_{s}(t)$ respectively. These functions can be identified only after transmission and delivery. Therefore the passivity condition in form of equation (5) cannot be obtained. However, the passivity condition can be conserved by keeping the decoupled forward and backward channel lines passive, as follows.

$$
\begin{aligned}
E_{c o m m, f w d} & =\frac{1}{2} \int_{0}^{t}\left[u_{m}^{2}(\tau)-u_{s}^{2}(\tau)\right] \mathrm{d} \tau \geq 0 \\
E_{c o m m, b w d} & =\frac{1}{2} \int_{0}^{t}\left[v_{s}^{2}(\tau)-v_{m}^{2}(\tau)\right] \mathrm{d} \tau \geq 0 .
\end{aligned}
$$

\section{Packet loss}

Using the Internet as the communication medium will induce occasional packet drops and therefore information loss in the exchange line, in addition to jitter (variations of delay). The same is valid for satellite based communication where the time delay function is smoother and the data loss rate is lower. Both mediums are discrete time systems where senders and receivers sample and pick the data with a certain frequency. An increase in time delay leads to the empty sampling instances for the receiver, which is usually called signal stretching in the continuous domain. The decrease in time delay leads to instances where more than one data packet exists to be sampled at the receiver site. This is called signal compression in continuous domain. However only one signal can be sampled and the other will be discarded. This leads to information loss. It is important to notice that the wave information has energetic meanings. Another source of packet-loss is the reordering of the sent signals where a later sent packet arrives earlier than an earlier sent packet. Typically empty sampling instances are solved by either inserting a zero value (Null packet or Zeroing strategy) or conveying the last valid signal, also known as Hold-last-sample (HLS). It has been shown that none of 
them is sufficient for performance or stability conditions and modification should be applied to them (Hirche and Buss, 2004). The Null packet strategy is lossy, overly conservative and leads to poor performance as well as wear and noise in mechanical parts. On the other hand, HLS strategy keeps the transmitted wave form well, but cannot guarantee the passivity of the channel and may lead to instability of the operation.

\subsection{Proposed method}

For the sake of simplicity the loss and time delay variations of the backward channel are neglected. Only the forward channel characteristics (dealing with backward link as ideal) are considered. After reformulating the continuous time expression in (6) for discrete time, the below relation is obtained.

$$
2 \Delta E(i) / T_{s}=\sum_{k=0}^{k=i} u_{m}^{2}(k)-\sum_{k=0}^{k=i} u_{s}^{2}(k),
$$

where $\sum_{k=0}^{k=i} u_{m}^{2}(k)$ is the sum of the square of the right moving wave variables and represents the power input to the forward communication block, $\sum_{k=0}^{k=i} u_{s}^{2}(k)$ is the sum of the received wave squares which is the power output of the communication and $T_{s}$ is the sampling time. The overall sent energy is calculated and its value is sent through the time delayed communication channel to the remote site (here slave). Hence, the remote receiver at time $t$ receives knowledge on the overall energy sent up to a certain time instance $\left(t^{*}\right) . t=n T_{s}$ is the current time and $t^{*}=n^{*} T_{s}$ is the time stamp of the arrived packet. These two times are related by :

$$
n T_{s}=n^{*} T_{s}+T_{f w d}\left(n^{*} T_{s}\right)
$$

To ensure the passivity, it is sufficient to state

$$
E_{\text {sent }}\left(n^{*} T_{s}\right)-E_{\text {received }}\left(n T_{s}\right) \geq 0 \text {. }
$$

Equation (10) is the online forward observed energy equation. The passivity of the channel has to be checked, comparing the overall energy input and output at the same time instances, but in practice the passivity condition cannot be measured online. Due to accumulative property of the sum of the sent wave energies, the following relation holds :

$$
E_{\text {sent }}(n) \geq E_{\text {sent }}\left(n^{*}\right) \text { where } n>n^{*}
$$


Considering the forward channel and renaming $E_{\text {sent }}$ by $E_{m}$ and $E_{\text {received }}$ by $E_{s}$, and based on (11), the following relation is valid

$$
E_{m}(n)-E_{s}(n) \geq E_{m}\left(n^{*}\right)-E_{s}(n) .
$$

The left hand side of (12) is the passivity condition and the right hand side is the online forward observed energy equation. Keeping the inequality in (10) leads to fulfilling the following overall passivity condition.

$$
E_{m}(n)-E_{s}(n) \geq 0
$$

The online forward observed energy equation is used for online passivity checks. As it is apparent, using this equation for passivity observation yields the system safety limits against the activity and the potential instability. A packet generator is implemented at each sender site putting the algorithm's required information into a data structure. The information put into the sending packets are sending time stamp $\left(n^{*} T_{s}\right)$, current sending wave value $\left(u_{m}\left(n^{*}\right)\right)$, packet reception acknowledgment flag, summation of the overall sent wave $\left(T_{s} \sum_{0}^{n^{*}} u_{m}\left(n^{*} T_{s}\right)\right)$ and the wave energy sent overall up to the current instance $\left(\frac{1}{2} T_{s} \sum_{0}^{n^{*}} u_{m}^{2}\left(n^{*} T_{s}\right)\right)$. The algorithm performs the following tasks to ensure the passivity. At each sampling time the packet reader on the receiver side checks the flag for arrival of data. When the flag indicates data arrival and its time stamp is bigger than the last recorded time stamp the packet data will be processed. This process consists of one-step-ahead energetic checks and if the online energy observer violated the passivity condition, the current wave is modified to dissipate required energy and keep the passivity. The program checks the theoretical energetical impacts if the current wave was conveyed using equation (14). $E_{\text {curr }}$ is the energy that the latest delivered wave could transmit to the system during one time sample.

$$
E_{\text {curr }}(n)=\frac{1}{2} u_{m}^{2}\left(n^{*}\right) T_{s}
$$

The forward energy observer $\left(E_{f e o}\right)$ checks the safety of conveying the current arrived wave in equation (15). $E_{\text {soutput }}(n-1)$ is the energy output of the packet processor up to the current time, which is fed back to the algorithm and $E_{\text {sent }}\left(n^{*}\right)$ is the sum of the energy sent from the master up to $n^{*}$ instance.

$$
E_{\text {feo }}(n)=E_{\text {sent }}\left(n^{*}\right)-E_{\text {soutput }}(n-1)-E_{\text {curr }}(n)
$$


If (15) is bigger or equal to zero, passivity would be kept by conveying $u_{m}\left(n^{*}\right)$. If (15) is negative, $u_{m}\left(n^{*}\right)$ has to be modified. In (17) the arrived $u_{m}\left(t^{*}\right)$ has to be changed in a way that dissipates the activity of $E_{f e o}(n)$. If $\hat{u}_{s}^{2}(n) \geq 0$ is satisfied, a real answer exists and using wave modification in one sample the sensed activity can be dissipated. We can refer to $u_{m}\left(n^{*}\right)$ as $u_{s}(n)$. The modification is applied to $u_{s}(n)$, based on the following energy balance equation.

$$
\frac{1}{2} u_{s}^{2}(n) T_{s}+E_{f e o}(n)=\frac{1}{2} \hat{u}_{s}^{2}(n) T_{s}
$$

Based on this, $u_{s}(n)$ has to be modified to $\hat{u}_{s}(n)$ following

$$
\hat{u}_{s}^{2}(n)=u_{s}^{2}(n)+\frac{2 E_{f e o}(n)}{T_{s}},
$$

to dissipate the $E_{f e o}(n)$ activity. The sign of the wave can be interpreted as push or pull command. Even though wrong signature selection does theoretically not affect the energetic behavior of the channel, the correct solution is important. This is more significant when a black-out occurs in the line and high number of consequent losses are detected. The trend of the sent command (push or pull) is observable by comparing the currently arrived sum of the sent waves with the last valid recorded arrived sum in (18). $n^{l v r}$ is the last-valid-recorded time instance of data arrival and is smaller than $n^{*}$.

$$
S=\sum_{i=0}^{i=n^{*}} u_{m}(i)-\sum_{i=0}^{i=n^{l v r}} u_{m}(i)
$$

When $S$ is positive or negative the trend indicates more push commands and more pull commands, respectively. Thus, the conveyed signal after a black out can be calculated.

$$
\hat{u}_{s}(n)=\frac{S}{|S|} \sqrt{u_{s}^{2}(n)+\frac{2 E_{f e o}(n)}{T_{s}}}
$$

For simple empty instances (due to reordering, occasional losses or stretching) selection of the signature, simply based on the current wave is correct but equation (19) generalizes these conditions as well.

If $\hat{u}_{s}^{2}(n)<0$ holds, the wave is replaced with a Zero, which is the maximum energy that can be dissipated in one instance. The remained undissipated energy is recorded to be dissipated in the next samples if necessary. As the activity detection in forward observed energy equation is conservative and preemptive and is based on the worst possible case (sending zero command 


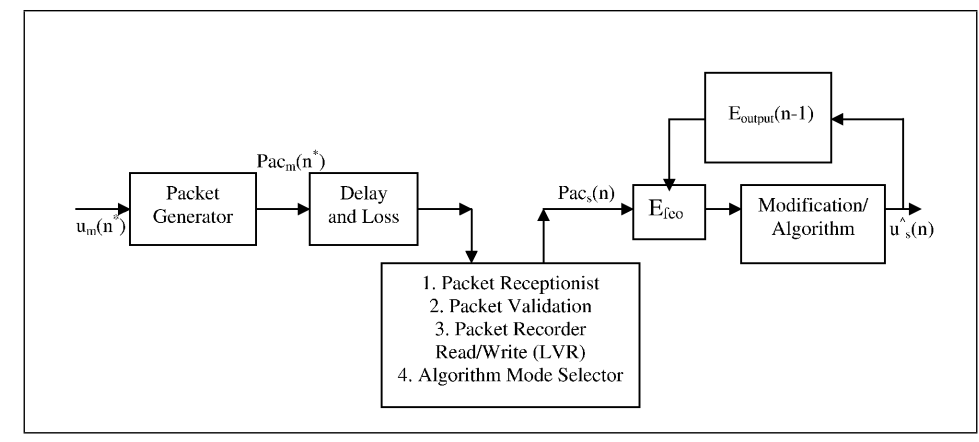

FIGURE 7 - Scheme of the forward link packet processing and energy compensation.

from counterpart), correction of these undissipated excess energy (remained after Zeroing) might not be necessary in the next steps. However, it is decided by the algorithm based on the received new data from the sender.

When the time stamp of the arrived packet is older than the last valid recorded time, the packet is discarded (The algorithm has already dealt with this packet's absence energetically as another later-stamped-earlier-arrived packet informed the receiver of the energy sent in between). There can be also instances that simply no packets are delivered. In both cases the algorithm has to handle the empty instance. The algorithm assumes that the current wave should be equal to the last valid signal (HLS strategy). With this assumption the HLS signal should be checked in the previous algorithm for energy considerations. If necessary the same modifications introduced before will be applied on the waves. The scheme of the proposed method and the integration into the forward link is depicted in Fig.7.

\section{Performance and results of the experiments}

An objective evaluation of telepresence is difficult since the feeling of being immersed into the remote environment is dependent on the subjective perception of the individual. The immersion of the user is influenced by the modality of the feedback from the teleoperator. The modality in turn depends on the properties of the link, which is used to transmit the feedback. Usually quality of service (QoS) criteria are used for evaluating terrestrial networks. Based on that, it has been shown (Chen, 2005; Park and Kenyon, 1999; Tfaily, 2003) that the QoS criteria round trip delay, jitter, and packet loss, in the context of space com- 
munications often expressed in terms of bit error rate can influence the task performance of a teleoperation.

The task performance of the human operator is directly related to the immersion of the human operator into the system, and thus a measure for telepresence. Out of the above criteria, the round trip delay is the one considered as most critical for teleoperation since time delay can destabilize a telepresence system

QoS criteria will initially be used for evaluating the telepresence capability of the system and related to the task performance of the human operator. For that purpose participants were asked to manipulate the OOS test bed under psychological instruction. The focus was laid on the task performance depending on the round trip delay characteristics of the space link, i.e. the progression of the round trip delay over time.

Finally, for a numerical determination of the telepresence capability of the system, an evaluation of the transparency of the system has been undertaken.

\subsection{Link characteristics depending task performance}

Using the mirror approach, the signal from the master manipulator at DLR traveled to the LRT ground station, was transmitted to ARTEMIS, forwarded to Redu, was mirrored, and was analogously redirected back to the DLR slave manipulator. According round trip delay characteristics were generated. The characteristic involves the behavior of the round trip delays over time. Round trip delay characteristics with a mean round trip delay of $\bar{t}_{R T D}=$ $622 \mathrm{~ms}$ were obtained. The characteristic in Fig. 8 shows a periodic behavior of the round trip delay. Every approximately 1000 packets the round trip delay increases abruptly and decreases slowly afterwards. This behavior is superimposed by fluctuations, featuring a smaller span. Additionally, high peaks (>2000 ms) occurred, which are caused by the comparable high amount of lost packets $(\approx 5.8 \%)$. This in turn originates mostly from errors in the satellite modem, as a lot of received packets had incorrect checksums and to some minor degree from the use of UDP in the connection with the terrestrial WAN, since the space link itself featured $<0.1 \%$ of lost packets. For evaluating the jitter of a signal, i.e. the variation in the round trip delay, it has been reverted to the sample standard deviation $s_{t}$ since the samples 
can be considered as statistically (quasi) independent. The sample standard deviation has been calculated to $s_{t}=66 \mathrm{~ms}$, which means that approximately $68.3 \%$ of all data samples are located in the interval $622 \mathrm{~ms} \pm 66 \mathrm{~ms}$.

Bit error ratios were measured within a dedicated BER test session. Usually the DRS service of ARTEMIS stops after an hour for a couple of minutes. The reason is that the SKDR payload has to be recalibrated. Thus, BER tests were usually limited to approximately one hour. However, ESA arranged an uninterrupted test session for 135 minutes, in which the BER could be measured. A BER of $<10^{-5}$ is regarded as standard acceptable for satellite telemetry and $<10^{-6}$ for the telecommand system. Thus the evaluated $8.3 * 10^{-6}$, can be considered as sufficient for satellite communication.

Large round trip delays in the haptic and visual channel worsen task performance and telepresence feeling (Pongrac, 2008). This effect is in particular more distinct if the round trip delays are varying. The tests, conducted within the framework of this work, second this fact.
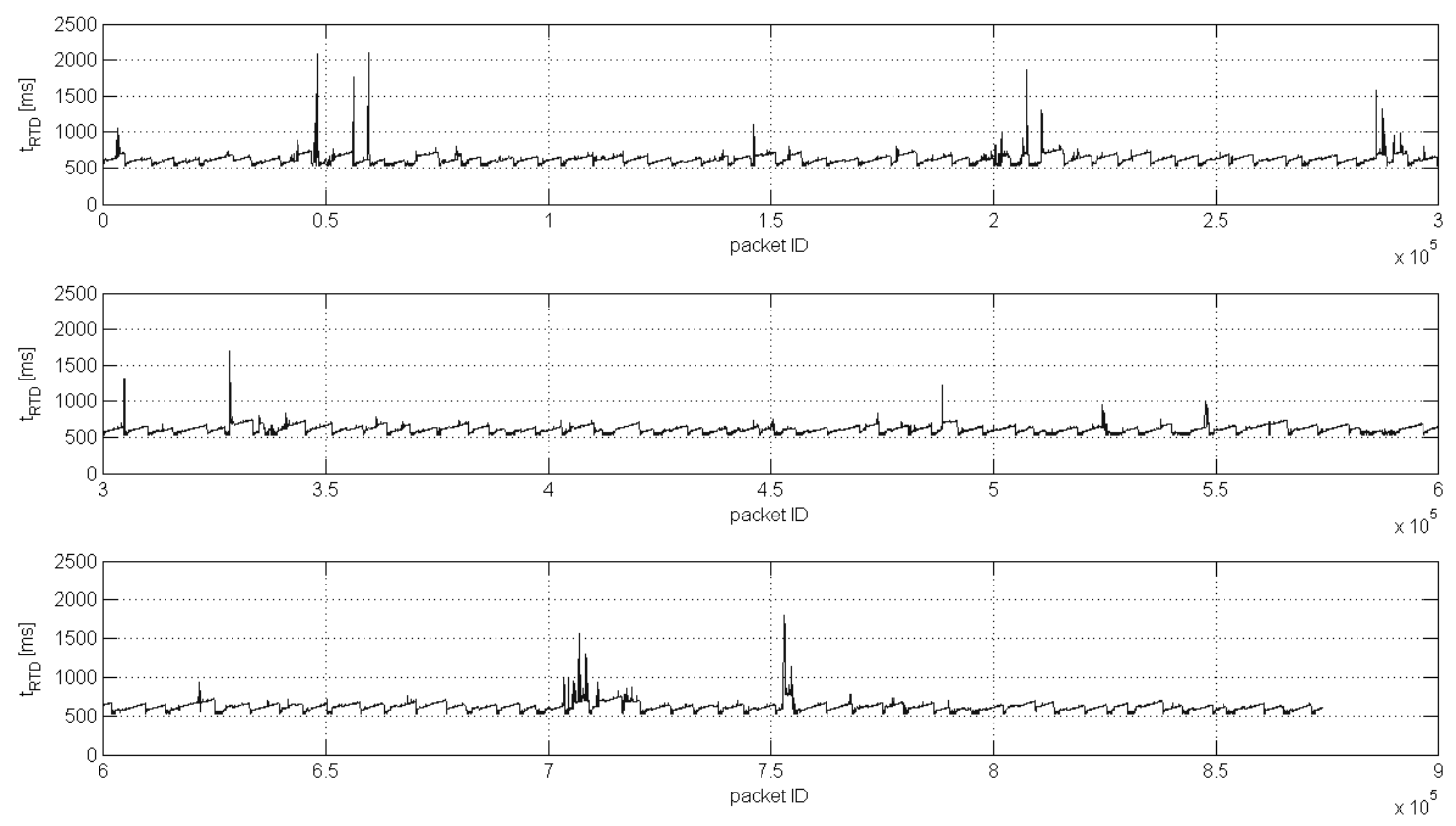

FIGURE 8 - Round trip delay characteristics of the telerobotic manipulation

Using DRS for teleoperation increases the round trip delay, which worsens the telepresence capability of a system in general. For an initial evaluation of the telepresence capability of the system a group of participants was asked to participate in a psychological evaluation, 
consisting of one exercise and two experimental trials. Because of the limited availability of the ARTEMIS link the group only consisted of six participants, who were completely unfamiliar with the system. Thus, the evaluation started with an exercise course. For becoming acquainted with the system, the bayonet nut connector (see Fig. 5) had to be opened several times by means of the robotic manipulator under 0 s round trip delay. The exercise was regarded as finished when the practice criterion was met, which was to fulfill the task within 30 s or in $25 \%$ of the time, needed in the first trial. Afterwards, the experimental trials started anew with 0 s round trip delay. The remote environment, i.e. the non-cooperative, malfunctioning satellite started moving and the participants had to grip it via the slave manipulator. Once the handhold on the test board was caught, the requirements for a successful docking operation were considered as met. The time for performing the first task was measured. The satellite was brought to initial position and no relative movement between manipulator and satellite was further introduced. The anew opening of the bayonet nut connector was the second task to be fulfilled. This is quite a complex task for a teleoperation, since the participants had to grip the respective part of the connector and execute a turn to release it from the second part. For a final disconnection of both parts, the extraction had to be fulfilled exactly parallel to the board. Otherwise the parts cant and a disconnection is not possible. The performance time was again logged.

The following second experimental trial was identical with the one, executed earlier (0 ms), except for the round trip delay. The two different tasks were performed using the ARTEMIS link and thus, with a comparably high and not constant round trip delay (see Fig. 8). The participants had to answer three questions after the experimental trials.

- How natural did the interaction with the environment feel (telepresence feeling) ? The scale ranged from 1 (very artificial) to 7 (very natural).

- How deeply did you feel immersed into the remote environment (immersion into the system)? The scale ranged from 1 (very weak) to 7 (very deeply).

- In your opinion, of what magnitude was the round trip delay? 
The task performance of the group of participants that was measured, is depicted in Fig. 9. The figure shows that the task performance of the human operator decreases due to the use of the data relay satellite. While the participants required (after a training phase) a mean of $15.0 \mathrm{~s}$ (with a sample standard deviation $\sigma=13.1 \mathrm{~s}$ ) to grip the satellite without time delay, using the master manipulator, the gripping time increased to $44.8 \mathrm{~s}(\sigma=23.0$ s) in the presence of the DRS. The mean time for opening the bayonet nut connector more than tripled from $31.0 \mathrm{~s}(\sigma=15.1 \mathrm{~s})$ to $111.6 \mathrm{~s}(\sigma=94.7 \mathrm{~s})$. Further, the results show that the participants systematically overestimate the round trip delay, even in the $0 \mathrm{~ms}$ case (estimated mean $226 \mathrm{~ms}$ ). By using the ARTEMIS link the participants estimated the round trip delay in mean to $952.6 \mathrm{~ms}(\sigma=587.0 \mathrm{~ms})$. The mean telepresence feeling (rating 1) dropped by 1.75 from $5.46(\sigma=1.38)$ to $3.71(\sigma=1.35)$, which corresponds from the psychological point of view a rating decrement of $214 \%$. The mean immersion into the system (rating 2) dropped by 0.55 points from $5.17(\sigma=1.52)$ to $4.62(\sigma=1.47 \mathrm{~s})$, which corresponds to a rating decrement of $130 \%$. Additional tests showed that there was no significant correlation between the demographical data of the participants (age, gender, handedness etc.) and the experimental data. Therefore, the logged values were most likely only depending on the experimental setup. Even though participants were small in number, the tests clearly showed that all participants were able to fulfill the assigned tasks via the

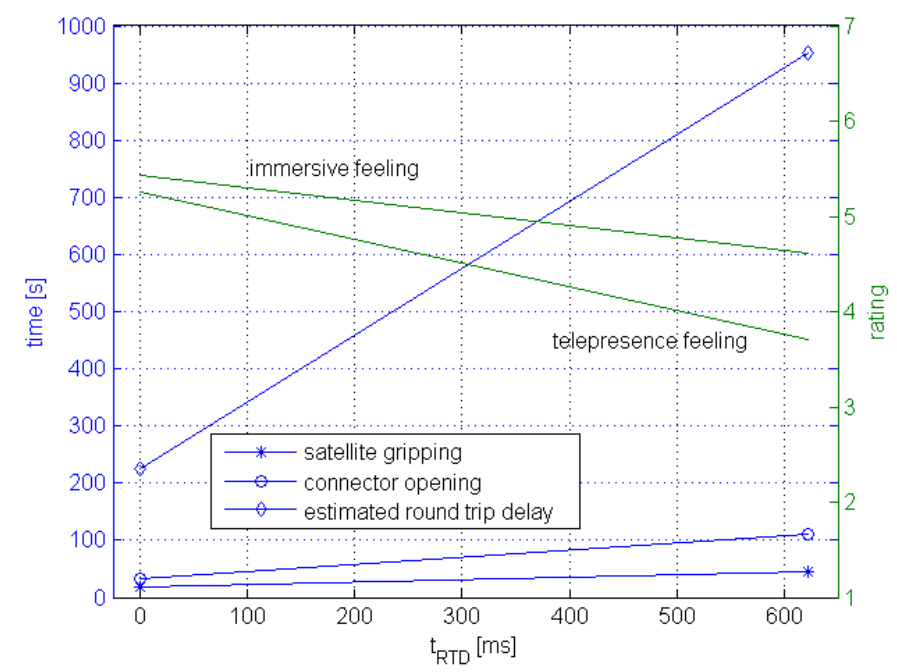

FIGURE 9 - Mean task performance of participants 
relay satellite in the presence of round trip delay. This will be the basis for additional tests.

\subsection{Transparency evaluation}

A haptic MMI can be approximated as a device, which generates mechanical impedance (Colgate and Brown, 1994). It represents the dynamic relationship between displacement (or velocity) and force. An ideal haptic interface would be capable of generating any impedance, which is required to represent the remote environment realistically to the human operator. Accordingly, transparency can be considered as the accuracy in rendering the remote environment to the human operator (Hashtrudi-Zaad and Salcudean, 2001).

After stability, the major goal of any haptic telepresence system is transparency. Previous works (Lawrence, 1993; Yokokohji and Yoshikawa, 1994) exhaustively show that the pursuit of stability compromises transparency once the system constraints are established. In particular, the presence of delay in the communication channel leads to a conservative design of the control architecture, lowering the system transparency and hence, the telepresence feeling in general. For a numerical confirmation of the above psychological evaluation of the teleoperation via ARTEMIS, a transparency evaluation of the master slave system was conducted, which was based on the haptic feedback channel. This evaluation was independently conducted from the psychological evaluation and did not require any participants. For evaluating the performance of a teleoperated system, the $Z$-width concept was used.

\subsection{The Z-width concept}

The Z-width is defined as the achievable range of impedance which the system can stably present to the operator (Colgate and Brown, 1994). Here the impedance to human $Z_{\text {toh }}(s)$ is defined by

$$
Z_{t o h}(s)=\frac{F_{h}(s)}{X_{m}(s)} .
$$

The Z-width range is delimited by frequency dependent lower and upper bounds. The lower bound of Z-width, $Z_{\min }(s)$, is calculated for $Z_{e}(s) \rightarrow 0$, while the upper bound, $Z_{\max }(s)$, is 
calculated for $Z_{e}(s) \rightarrow \infty$. That is

$$
\begin{aligned}
& \text { Free Motion : } \quad Z_{\text {min }}(s)=\left.Z_{\text {toh }}(s)\right|_{Z_{e}(s) \rightarrow 0} \\
& \text { Constrained Motion : } Z_{\max }(s)=\left.Z_{t o h}(s)\right|_{Z_{e}(s) \rightarrow \infty} \text {. }
\end{aligned}
$$

Consequently, the Z-width is given by

$$
Z_{\text {width }}(s)=Z_{\max }(s)-Z_{\min }(s)
$$

This Z-width allows to compare different control schemes quantitatively. Since the bandwidth of human actuation and sensing capabilities are limited (Lawrence et al., 2004), the analysis of the Z-width in this paper will be restricted to the relevant frequency range.

\subsection{Z-width measurement procedure}

If the human force and master position can be measured, then the human perceived impedance can be obtained from these measured signals using Least-Squares Input/Output (LS I/O) Identification method (Hirche et al., 2003). The goal of the identification method is to find the parameters of the transfer function of the human perceived impedance that minimize the squared error between the real output and the output of the identified transfer function. The following procedure is followed in order to measure the Z-width out from the measured human percieved impedance :

1. Measurement of the human operator force $f_{h}$ and the master position $x_{m}$ in the free movement case.

2. I/O system identification of the transfer function $Z_{t o h}=\frac{f_{h_{\text {free }}}}{x_{m_{\text {free }}}}$. This is the measured minimum limit of the Z-Width, $\hat{Z}_{\text {min }}$.

3. Measurement of the human operator force $f_{h}$ and the master position $x_{m}$ in the case of wall contact.

4. I/O system identification of the transfer function $Z_{\text {toh }}=\frac{f_{h_{\text {wall }}}}{x_{m_{\text {wall }}}}$. This is the measured maximum limit of the Z-Width, $\hat{Z}_{\max }$.

5. The measured Z-Width, $\hat{Z}_{\text {width }}$, is the difference between the two identified impedances $\hat{Z}_{w i d t h}=\hat{Z}_{\max }-\hat{Z}_{\min }$. 
Note that this evaluation was only done in 1-dimensional space for obtaining scalar values for force and displacement. The two cases free movement and wall contact were generated by randomly moving the slave in free space and by moving the slave into a rigid wall (satellite), respectively. The progression of master displacement $x_{m}$ and slave displacement $x_{s}$ (in the time domain) is illustrated in Fig. 10. The position of the slave (dashed) follows the master (solid) after a round trip delay, which is caused by the relay via the DRS. A wall is located at zero position. The free environment is indicated by a negative sign of the displacements. It can be seen that initially a movement in free environment has been conducted, followed by a movement into the virtual wall. The master displacement at that point in time becomes positive, whereas the slave motion is constrained by the wall. Thus, its displacement is al-

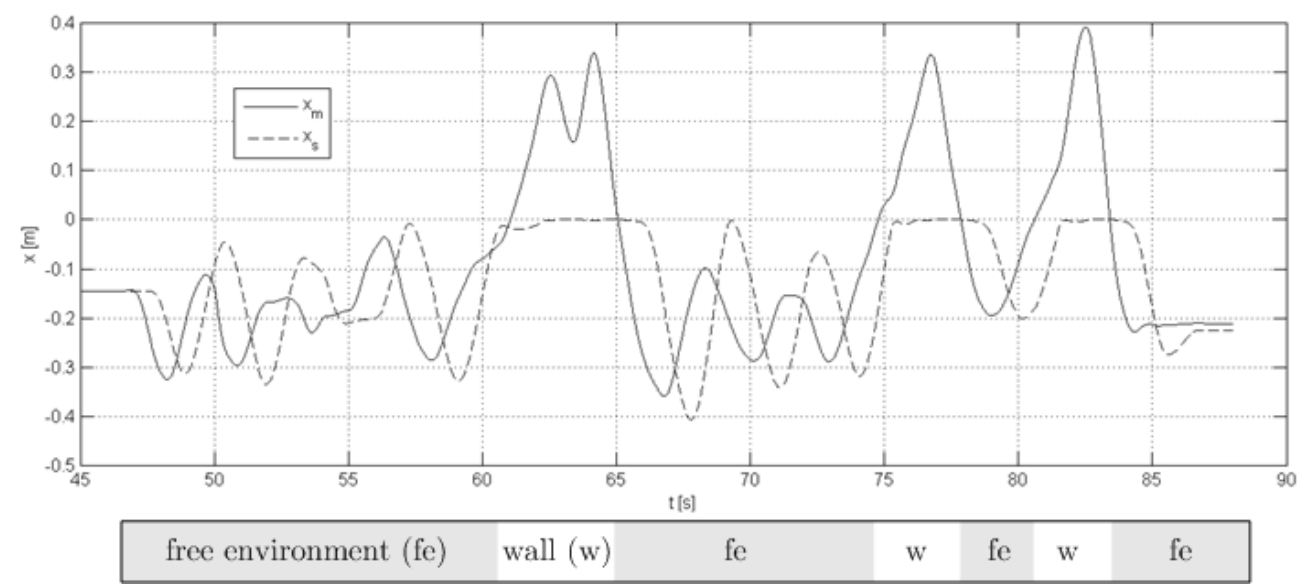

FIGURE 10 - Displacement of master and slave over time

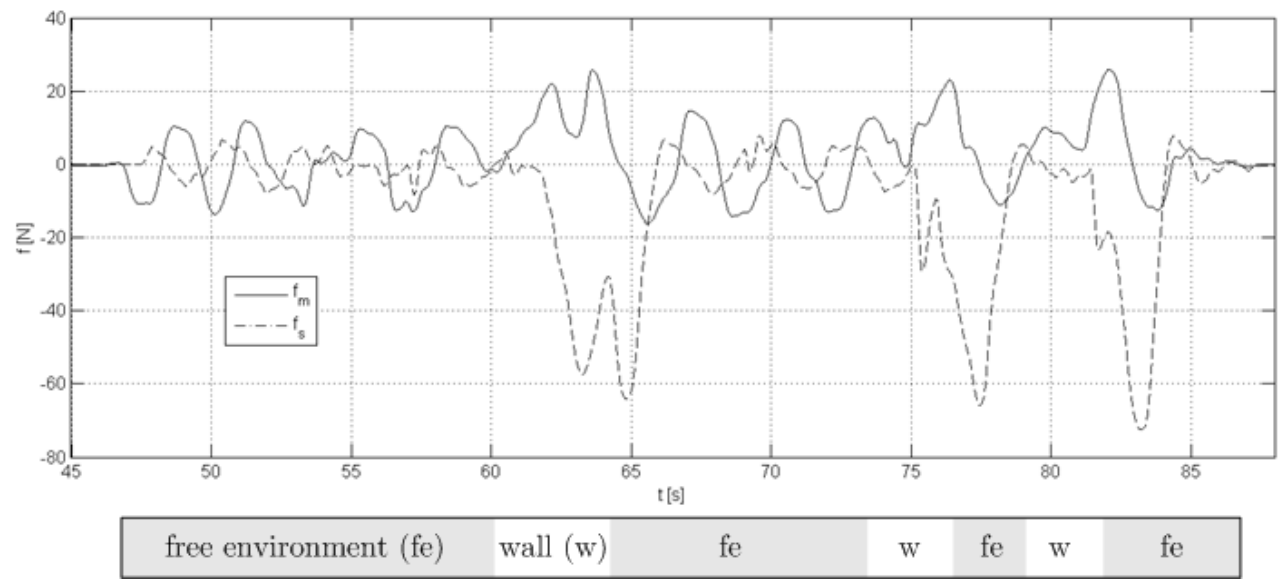

FIGURE 11 - Force of master and slave over time 
most constant at zero position.

Fig. 11 shows master and slave force in the time domain. The slave force is delayed by the DRS and features an opposite sign compared to the master force. Their absolute value is approximately identical in free environment and the absolute value of the slave force tends to be smaller. In contrast, for the wall condition the slave force increases to a multiple of the master force, considering absolute values. The values obtained that way were transferred

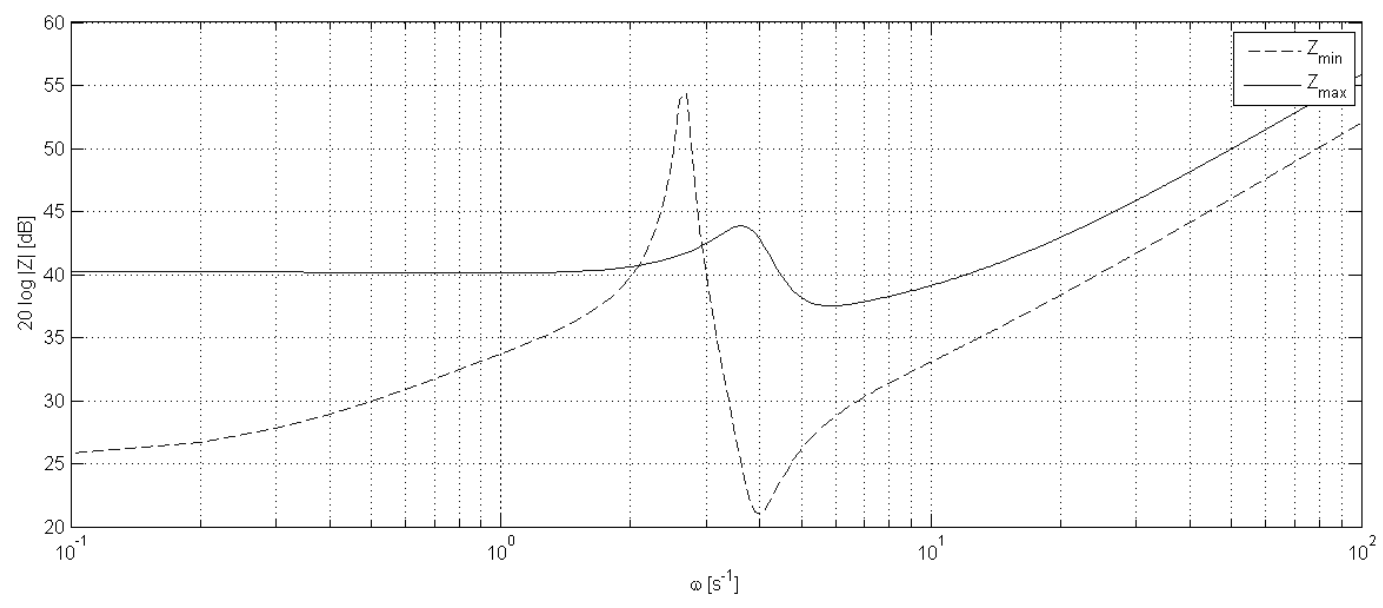

FIGURE 12 - Absolute values for $Z_{\min }$ and $Z_{\max }$ via ARTEMIS

into the frequency domain. $Z_{\min }$ and $Z_{\max }$ were derived and can be accessed from Fig. 12. Since the impedances are complex values, they were plotted over the frequencies using a 20 $\log |Z|$ scale to be compliant with a Bode representation. For approximating the transfer functions in Fig. 12 appropriate time intervals for wall contact and free environment from Fig. 10 and Fig. 11 were selected.

The corresponding $Z_{\text {width }}$ is depicted in Fig. 13. The Z-width concept is not an absolute indicator for system transparency. It rather can be utilized to evaluate the system transparency with respect to another system setup. For that reason the reference $Z_{\text {width }}$ for $0 \mathrm{~ms}$ is also plotted in Fig. 13. It can be seen that the increasing delay decreases the system transparency (in the low frequency range), analogously to the task performance of the human operator. This is compliant with theory. In the low frequency range the graphs can be assumed to be constant as a first approximation. It follows a peak (for the graphs with DRS), which is not of practical relevance because of the limits of the approximation. 
This limits originate from the fact that the Z-width is commonly derived by using a Padé series of finite order $N$ to describe the time delay system element $D_{t}$. By using a first order series $(N=1)$

$$
D_{t}=e^{-s T} \approx \frac{1-\frac{T}{s}}{1+\frac{T}{s}},
$$

the Padé approximation is only valid for frequencies $\omega_{l i m}<1 /(3 T)$ (Hirche et al., 2005). Therefore, the peaks are of no practical relevance, since the Z-width graph is only valid for the low frequency range.

The system frequency assigned to the axis of abscissa can be interpreted as frequency, with which the human operator steers the master manipulator. This is constrained by the limiting frequency and amounts in the given example to $\omega_{\text {lim }} \approx 0.5 \mathrm{~Hz}$. This limitation is of practical interest since it labels a region, in which the system cannot react fast enough anymore, due to the occurring round trip delay, to a given input. Accordingly, the user must not be allowed (by technical means, as e.g. inertia or damping) to execute teleoperations above this limiting frequency.

\section{Summary and future directions}

This work focused on utilizing the concept of telepresent control to on-orbit servicing. In particular, the applicability to OOS missions in low Earth orbit, for which the communi-

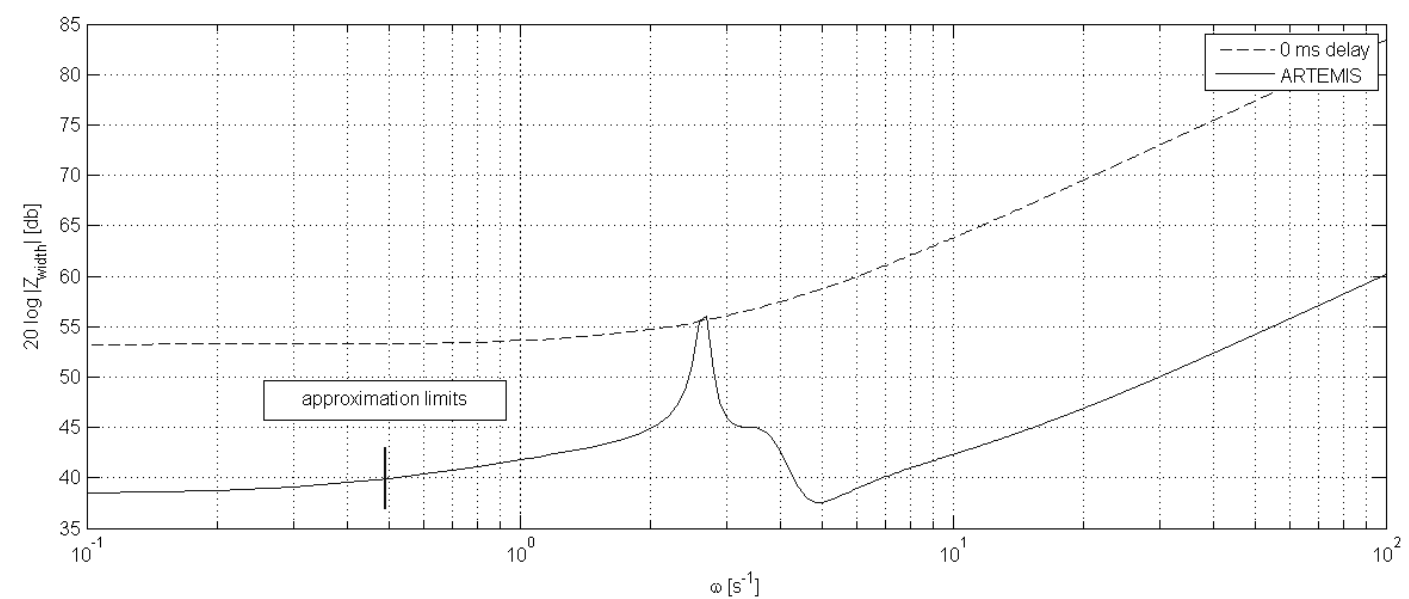

FIGURE 13 - Absolute value of the Z-width for zero delay and via ARTEMIS 
cation has to be relayed via a DRS in geostationary orbit, was considered. This section summarizes the results and gives suggestions for future work.

\subsection{Summary}

The term telepresence describes a control concept, in which a teleoperator is separated from the controlling human operator by a barrier. Telepresence demands that the human operator can hardly differentiate, whether his impressions of feedback result from direct interaction with the environment or from technical means.

Utilizing telepresence for OOS operations promises a number of advantages for astronautics. On the one hand, the human operator is located on ground and thus, cost intensive and critical EVAs can be avoided. On the other hand, it enables the human operator a real time response to unforeseen incidents, which is not possible considering autonomous OOS missions.

Contrary to terrestrial applications, the contact time to the teleoperator is limited, especially in LEO. Direct contact from the ground station to the servicing spacecraft is only given for a few of minutes, which limits the data acquisition time. Hence, the use of geostationary satellites for data relay was considered, which increases the acquisition time to a multiple, compared to direct contact. However, the disadvantage of this approach is the increase of round trip delay, jitter and package loss.

It could be shown that using the appropriate bilateral control strategy this increase in round trip delay and non-idealities does not conflict with the concept of telepresence control, for which a realistic feedback is demanded to some extend. A test environment was developed, which was representative for telepresent OOS using a DRS and involved the Institute of Astronautics, the European Space Agency, and the German Aerospace Center. A robotic OOS test bed was teleoperated via the ESA satellite ARTEMIS. The implementation of ARTEMIS and the setup on ground enabled obtaining realistic measurements, in order to evaluate the feasibility of telepresent OOS.

The obtained round trip delays with a mean of $622 \mathrm{~ms}$ enabled an accomplishment of the 
telepresent manipulation tasks, even under the influence of additional network delays. For validating the above statement, a psychological evaluation was conducted. The feeling of telepresence or immersion into the system is a subjective perception. Thus, a number of participants were asked to steer the robotic scenario via ARTEMIS. The results show that it is possible to execute complex OOS maneuvers via a geostationary DRS and maintain the feeling telepresence. This was corroborated by an evaluation of the system transparency. Based on that, the conclusion is drawn that telepresent on-orbit servicing with haptic feedback via a geostationary relay satellite is feasible. Nonetheless, the number of participants was limited due to the availability of the satellite link. Thus, further testing will be needed to obtain a comprehensive human-machine evaluation.

\subsection{Future directions}

Network delays are part of the considered round trip delays. They can be minimized, when realizing a telepresent space mission, either by locating the human operator in proximity to the ground station or prioritizing the network link.

Future research has to consider the synchronization of system elements. The clock frequency of signal generating equipment and the communication equipment (e.g. IMBU) has to be adjusted with respect to each other in order to avoid peaks in round trip delay. The necessity of system buffers and a sufficient buffer management will be of further interest. Optimizing the control parameters of the system holds even more potential for enhancing the system transparency than a minimized round trip delay. The master-slave control architecture has to be adapted to the respective OOS operation to increase the telepresence capability.

By steering the robotic application via the DRS, the S-band link met its limits. The master sample rate had to be decreased to $500 \mathrm{~Hz}$ instead of the common $1000 \mathrm{~Hz}$. The stereo feedback (telemetry) had to be transmitted locally. Higher band width is indispensable for space missions. The Ka-band frequency range is a possible alternative. This would also allow transmitting additional sensor data from the haptic-visual work space, which enhances the telepresence capability of the system. This in turn will support the immersion of the human 
operator into the system.

\section{Acknowledgments}

This work is supported in part by German Research Foundation (DFG) within the collaborative research center SFB453 "High-Fidelity Telepresence and Teleaction". The authors would like to express their sincere gratitude to the ESA ARTEMIS team for providing the opportunity to use the ARTEMIS relay satellite for telepresence experiments. In particular, special thanks to Benoit Demelenne and Damien Dessoy for prompt help and unbureaucratic scheduling concerning the data relay service. Further, we would like to thank LSE Space Engineering, DLR MORABA, Rhode \& Schwarz, and DOMO TV for supporting the development of the LRT ground station.

\section{References}

AFRL (2007). Xss-11 micro satellite - fact sheet. Technical report.

Anderson, R. and Spong, M. (1989). Bilateral control of teleoperators with time delay. Automatic Control, IEEE Transactions on, 34(5) :494-501.

Artigas, J., Kremer, P., Preusche, C., and Hirzinger, G. (2006). Testbed for telepresent on-orbit satellite servicing. In in Proceedings of the Human-Centered Robotic Systems Conference (HCRS), Munich, Germany.

Artigas, J., Preusche, C., and Hirzinger, G. (2007). Time domain passivity for delayed haptic telepresence with energy reference. In proc. IEEE/RSJ International Conference on Intelligent Robots and Systems (IROS'07)., San Diego, CA, USA.

Artigas, J., Preusche, C., Hirzinger, G., Borghesan, G., and Melchiorri, C. (2008). Bilateral energy transfer in delayed teleoperation on the time domain. In proc. of IEEE/RSJ International Conference on Robotics and Automation (ICRA'08), Pasadena, CA, USA.

BOEING (2007). Orbital express - mission updates. Technical report.

Bosse et al., A. (2004). Sumo : spacecraft for the universal modification of orbits. In proc. of SPIE - The International Society for Optical Engineering, vol. 54 iss.19, Aug. 2004.

Chen, L. (2005). Effects of network characteristics on task performance in a desktop cve system. In proc. of 19th IEEE International Conference on Advanced Information Networking and Applications, Tamkang, Taiwan, Mar. 2005.

Colgate, J. and Brown, J. (1994). Factors affecting the z-width of a haptic display. In Robotics and Automation Proceedings, IEEE International Conference on, pages 3205-3210 vol.4.

Davis, T. (2005). Xss-10 micro-satellite flight demonstration. In proc. of Georgia Institute of Technology Space Systems Engineering Conference, paper no. GT-SSEC.D.3, Nov. 2005. 
Hannaford, B. and Ryu, J.-H. (2000). Time Domain Passivity Control of Haptic Interfaces. IEEE Transactions on Robotics and Automation.

Hashtrudi-Zaad, K. and Salcudean, S. E. (2001). Analysis of control architectures for teleoperation systems with impedance/admittance master and slave manipulators. The International Journal of Robotics Research, 20(6) :419-445.

Hirche, S., Bauer, A., and Buss, M. (2005). Transparency of haptic telepresence systems with constant time delay. In proc. of IEEE Conference on Control Applications, Toronto, Canada, 2005.

Hirche, S. and Buss, M. (2004). Packet loss effect in passive telepresence systems. In 43rd IEEE Conference on Decision and Control, pages 4010-4015.

Hirche, S., Stanczyk, B., and Buss, M. (2003). Transparent exploration of remote environments by internet telepresence. In proc. of the International Workshop on High-Fidelity Telepresence and Teleaction jointly with the IEEE conference HUMANOIDS, (Munich, Germany), 2003.

Hirzinger, G., Landzettel, K., Brunner, B., Fischer, M., Preusche, C., Reintsema, D., AlbuSchäffer, A., Schreiber, G., and Steinmetz, M. (2004). DLR's robotics technologies for on-orbit servicing. Advanced Robotics - Special Issue Service Robots in Space, 18(2) :139174.

Imaida, T., Yokokohji, Y., Doi, T., Oda, M., and Yoshikawa, T. (2004). Ground-space bilateral teleoperation of ets-vii robot arm by direct bilateral coupling under 7-s time delay condition. Robotics and Automation, IEEE Transactions on, 20(3) :499-511.

Landzettel, K., Preusche, C., Albu-Schäffer, A., Reintsema, D., Rebele, B., and Hirzinger, G. (2006). Robotic On-Orbit Servicing - DLR's Experience and Perspective. In proc. of the International Conference on Intelligent Robots and Systems (IROS), Peking, China.

Lawrence, D. (1993). Stability and transparency in bilateral teleoperation. Robotics and Automation, IEEE Transactions on, 9(5):624-637.

Lawrence, D., Pao, L., White, A., and Xu, W. (2004). Low cost actuator and sensor for highfidelity haptic interfaces. In Haptic Interfaces for Virtual Environment and Teleoperator Systems, 2004. HAPTICS '04. Proceedings. 12th International Symposium on, pages 74-81.

Lundin, R. and Stoll, E. (2006). Coverage time variation in a near-earth data relay satellite system. In 57th International Astronautical Congress, Valencia, Spain, Oct. 2006.

Moens, C., Absolonne, F., and Lezy, C. (2003). Artemis data relay payload iot results as measured from redu in feb/mar 2003. Technical report.

Mukherji, R., Rey, D., Stieber, M., and Lymer, J. (2001). Special purpose dexterous manipulator (spdm) advanced control features and development test results. In proc. of the 6th International Symposium on Artificial Intelligence and Robotics and Automation in Space (i-SAIRAS), St-Hubert, Canada, Jun. 2001.

Niemeyer, G. (1996). Using Wave Variables in Time Delayed Force Reflecting Teleoperation. PhD thesis, Massachusetts Institute of Technology, Department of Aeronautics and Astronautics Engineering, Cambridge, MA.

Oda, M. (2000). Experiences and lessons learned from the ets-vii robot satellite. In Robotics and Automation, 2000. Proceedings. ICRA '00. IEEE International Conference on, volume 1, pages 914-919 vol.1. 
Park, K. and Kenyon, R. (1999). Effects of network characteristics on human performance in a collaborative environment. In proc. of IEEE International Conference on Virtual Reality, Houston, USA, Mar. 1999.

Parrish, J. and Akin, D. (1996). The ranger telerobotic flight experiment : Mission, technologies, and programmatics. In proc. of Conference on Robotics for Challenging Environments, Albuquerque, USA, Jun. 1996.

Peters II, R. and Campbell, C. (1999). Robonaut task learning through teleoperation. In proc of IEEE Inter-national Conference on Robotics and Automation, Taipei, Taiwan, September, 2003.

Pongrac, H. (2008). Gestaltung und Evaluation von virtuellen und Telepräsenzsystemen an Hand von Aufgabenleistung und Präsenzempfinden. PhD thesis, Human Factors Institute, Universität der Bundeswehr, 2008.

Preusche, C., Reintsema, D., Landzettel, K., and Hirzinger, G. (2006). ROKVISS - Preliminary Results for Telepresence. In proc. of the International Conference on Intelligent Robots and Systems (IROS), Peking, China.

Rumford, T. (2003). Demonstration of autonomous rendezvous technology (dart) project summary. In proc. of Space Systems Technology and Operations Conference, Orlando, USA, Apr. 2003.

Sommer, B. (2003). Automation and robotics in the german space program - unmanned onorbit servicing (oos) and the tecsas mission. In proc. of 55th International Astronautical Congress, Vancouver; Canada, Oct. 2004.

Stoll, E. (2008). Ground Verification of Telepresence for On-Orbit Servicing. PhD thesis, Institute of Astronautics, Technische Universität München, 2008.

Tarabini et al., L. (2007). Ground guided cx-olev rendezvous with uncooperative geostationary satellite. Acta Astronautica; Selected Proceedings of the 57th IAF Congress, Valencia, Spain, Oct. 2006, 61(1-6).

Tfaily, R. e. a. (2003). Haptic virtual environment performance over ip networks : A case study. In proc. of 7th IEEE International Symposium on Distributed Simulation and Real-Time Applications, Delft, The Netherlands, Oct. 2003.

Yokokohji, Y. and Yoshikawa, T. (1994). Bilateral control of master-slave manipulators for ideal kinesthetic coupling-formulation and experiment. Robotics and Automation, IEEE Transactions on, $10: 605-620$. 